\title{
A escolha do método contraceptivo
}

\author{
Contraceptive method choice
}

Luis Bahamondes ${ }^{1}$

O artigo de Matarezi de Souza et al. ${ }^{1}$ realizado no estado do Paraná avalia a situação da utilização de métodos contraceptivos (MACs) entre usuárias atendidas por 62 equipes do Programa da Saúde da Família. Os dados foram obtidos mediante entrevistas com 284 mulheres. Em minha opinião os dados mais relevantes que os autores encontraram foram que a prevalência de MACs no município estudado concentra-se em mais de $80 \%$ dos casos em usuárias de apenas dois métodos: o anticoncepcional oral, responsável por $50 \%$ dos casos, e a ligadura tubária, presente em quase um terço dos mesmos. Além disso, apenas 2,1\% declararam estar usando dispositivo intra-uterino (DIU), e o uso de injetável trimestral e mensal foi desprezivel. Isto não deveria surpreender o leitor, já que os achados refletem a realidade observada previamente em outros inquéritos populacionais realizados em outras partes do Brasil ${ }^{2,3}$.

Este artigo mostra, também, que um terço das mulheres usuárias de anticoncepcional oral iniciou o uso da pílula sem nenhuma consulta médica prévia e, talvez por este motivo, aproximadamente metade das usuárias apresentou alguma contra-indicação para seu uso, segundo os critérios de elegibilidade estabelecidos pela Organização Mundial da Saúde (OMS) ${ }^{4}$. O que chama a atenção nestes resultados é que menos de $10 \%$ das entrevistadas pertenciam às classes econômicas A e B e que apenas $7,7 \%$ delas tinham como grau de escolaridade o primeiro grau incompleto.

Estes comentários apontam o que é conhecido: com maior escolaridade e melhor nivel socioeconômico, as mulheres têm melhor conhecimento e acesso a um leque mais amplo de MACs $^{2,3}$. Assim, era de se esperar que estes resultados mostrassem que estas mulheres apresentavam maior prevalência de uso de outros MACs diferentes da pílula e da laqueadura, fato não observado neste estudo. Entretanto, isto poderia ser reflexo de que pouco mais da metade destas mulheres não exercia nenhum trabalho remunerado, o que provavelmente levou a um acesso restrito às alternativas de métodos contraceptivos, o que reflete a situação no setor público da saúde no tocante ao planejamento familiar.

A Constituição do Brasil de 1988 estabelece que homens e mulheres tenham direito ao uso de métodos contraceptivos e que o Estado tem a obrigação de fornecer os mesmos. Entretanto, os resultados deste estudo mostram que na prática o planejamento familiar no Brasil se apóia quase que exclusivamente nos dois métodos observados, a pílula e a ligadura tubária ${ }^{2,3}$, sendo muito restrito o acesso aos outros métodos.

Poderíamos concluir que isto não seria um problema, já que, se a meta é que as mulheres em idade reprodutiva tenham acesso a métodos contraceptivos quando o desejarem, os resultados do estudo mostraram que a grande maioria era usuária de um MAC moderno e de alta eficácia. Isto vai ao encontro da observação da alta prevalência de uso de MACs entre mulheres brasileiras 5 . Entretanto, os resultados deveriam ser interpretados, ao meu ver, de uma forma diferente.

Um dos fatos preocupantes é que, entre as usuárias de contraceptivos orais, quase metade tinha recebido o seu método sem nenhuma consulta médica prévia, como mencionado. Isto é resultado do fato de que na grande maioria dos locais de atenção médica o planejamento familiar não tem sido entendido como ação de medicina preventiva. As agendas de consultas médicas são habitualmente lotadas, levando muitas mulheres ao desanimo com o atendimento no setor público, preferindo adquirir contraceptivos orais diretamente com o "doutor do balcão", onde os contraceptivos orais e injetáveis, e outros medicamentos fornecidos sob receita, são vendidos como se fossem de venda livre.

1 Professor Titular do Departamento de Tocoginecologia Faculdade de Ciências Médicas - Universidade Estadual de Campinas (UNICAMP) Campinas (SP), Brasil. 
Além das agendas médicas lotadas que dificultam o acesso das mulheres à atenção médica, a falta de acesso também é conseqüência da não-incorporação de outros profissionais na atenção de consultas de contracepção, novamente porque não se tem interpretado a anticoncepção como medicina preventiva.

Este estudo mostrou que em torno de metade das usuárias apresentavam algum fator de risco ou alguma contra-indicação ao uso de contraceptivos orais. Este fato já foi observado há muito tempo em inquérito realizado no estado de São Paulo durante uma campanha de vacinação contra a poliomielite: também metade das mulheres usuárias de contraceptivos orais entrevistadas e avaliadas apresentavam algum fator de risco para seu uso $^{6}$. Além disto, 17,4\% estavam em alto risco de complicações para o uso de contraceptivos orais, o que mostrou o baixo conhecimento da população sobre os problemas da associação de contraceptivos orais com o fumo e hipertensão arterial. Os resultados daquele estudo e do presente apontam para a falta de informação entre usuárias.

Este fato é conseqüência, novamente, da automedicação e do fato de se poder adquirir os contraceptivos diretamente sem receita e sem nenhuma avaliação prévia. Entretanto, não queremos passar a idéia de que tomar anticoncepcionais orais combinados seja prática de alto risco, porém, devemos estar atentos aos fatores de risco preexistentes ou que aparecem durante o uso.

Devido ao fato de os profissionais médicos estarem saturados de consultas, devemos incorporar outros profissionais, como os médicos do Programa de Saúde da Família e os enfermeiros, os quais podem ser de grande valor na ajuda da implementação das ações de planejamento familiar.

Uma forma de atuação seria a aplicação de listas verificadoras (check-lists) existentes e em uso em muitos países. Desta forma, com o uso de um questionário pode-se avaliar a presença dos fatores de risco mais importantes para restringir o uso de contraceptivos orais e injetáveis, liberando os médicos de centenas ou milhares de consultas não necessárias e permitindo, desta forma, concentrar-se naquelas mulheres que realmente precisam de sua atenção ${ }^{7}$.

Ainda quanto à distribuição de métodos contraceptivos foi observada alta prevalência de mulheres já laqueadas, embora o trabalho não permita discriminar a sua idade nem a idade em que foi realizada a cirurgia. Este fato já é conhecido por outros inquéritos prévios. Entretanto, este dado tem ficado restrito ao âmbito acadêmico, mas é necessário que seja conhecido pela população e principalmente pelos ginecologistas aos quais está destinada esta revista.

As mulheres recorrem à laqueadura por falta de opções contraceptivas ou porque pensam que este método nunca falha. Não recebem informações adequadas sobre os outros MACs, não têm acesso aos mesmos ou não conhecem as reais taxas de falha. Por outro lado, os casais se separam e voltam a constituir novos casais, desejando um novo filho. Isto resulta em número crescente de mulheres arrependidas que solicitam reversão da cirurgia nos serviços especializados de esterilidade, como tem acontecido em nosso serviço ${ }^{8,9}$.

Inquérito da década dos anos 80 na UNICAMP mostrou que $2,3 \%$ das consultas novas de esterilidade eram de mulheres que solicitavam reversão da cirurgia. Para a década de 90 e 2000 estas cifras se elevaram para quase $14 \%$, o que mostra que algo deve ser realizado para informar melhor as mulheres antes de serem submetidas a uma cirurgia, que na maioria dos casos não tem possibilidades de reversão ${ }^{10}$. Nestes casos, resta somente a angústia ou a resignação, já que a maioria destas mulheres não terá possibilidades de engravidar por meio de procedimento de fertilização assistida.

Além disso, as mulheres optam pela laqueadura porque argumentam que não desejam mais filhos, acrescentando que a utilização de anticoncepcionais orais faz mal ou por referirem intolerância aos mesmos. Novamente aparecem os contraceptivos orais como a única opção à ligadura tubária, não havendo a possibilidade de optar por outros MACs. Tem-se observado que a menor idade no momento da cirurgia é o fator mais importante de risco para solicitação de reversão da laqueadura ${ }^{11,12}$, seguido do menor conhecimento de outros métodos contraceptivos que sejam de alta eficácia e ao mesmo tempo reversíveis.

Como muitas mulheres são submetidas a cirurgia em idade muito precoce, por falta de opção, eleva-se o risco de solicitação de reversão por divórcio do casal e nova união com desejo de um novo filho, por falecimento de um filho, por melhoria da condição econômica, entre outras razões que podem levar ao arrependimento.

É conhecido por estudos realizados nos Estados Unidos da América ${ }^{13}$ que a taxa de falha da laqueadura é em torno de 0,18 por 100 cirurgias ou quase duas gravidezes por cada 1000 mulheres laqueadas, mas estas cifras podem ser maiores, dependendo da técnica cirúrgica empregada. Por 
que fazer laqueadura se existem outros MACs de igual eficácia e que são reversíveis? A pergunta não tem uma única resposta. Isto ocorre por falta de opções, por falta de acesso, por má informação ou por temor ao uso de alguns métodos, entre outras causas.

Existem excelentes métodos contraceptivos, como o injetável trimestral com acetato de medroxiprogesterona de depósito, os injetáveis mensais combinados, os implantes contraceptivos subdérmicos, o sistema intra-uterino liberador de levonorgestrel e o DIU com cobre, todos com taxas de falha similares ou ainda menores que a laqueadura ${ }^{14}$, com a vantagem que todos são reversiveis e, conseqüentemente, permitem nova gravidez.

O DIU, que aparece neste estudo com uma prevalência tão baixa (apenas 2\%), é um excelente método que poderia ter sido aceito por um número maior de mulheres. O mais conhecido é o TCu380A, que está aprovado pelo Ministério da Saúde para uso, sem troca, durante dez anos. A taxa de falhas é de 0,3 por 100 mulheres/ano, o que significa que três mulheres ficarão grávidas em 1.000 usuárias, cifra muito parecida à da laqueadura ${ }^{15}$. Por outro lado, já existem dados da OMS segundo os quais o mesmo foi utilizado por até 12 anos sem troca e sem que houvesse redução no efeito contracepti$\mathrm{vo}^{16}$. Além deste, estudo recente realizado na UNICAMP avaliou o uso deste DIU por até 16 anos sem troca em mulheres com mais de 35 anos de idade ao momento de completar os 10 anos de uso. Os dados mostraram que não houve nenhuma gravidez o que permitiu estabelecer a hipótese de que este DIU poderia ser utilizado até à menopausa sem troca do mesmo, em mulheres que tiverem 25 anos de idade ou mais no momento da inserção ${ }^{17}$.

Gual seria a vantagem desta prática? O custo de um DIU, incluído os gastos com a inserção, são muito baixos, oferecendo proteção por longo tempo. Entretanto, esta baixa prevalência observada poderia refletir a falta do insumo, a falta de capacitação dos profissionais na técnica de inserção, a pobre orientação que as mulheres recebem em relação ao método, novamente por falta de profissionais capacitados, os mitos de usuárias e profissionais ou a restrição de uso por alguns profissionais ou uma conjunção de todas as causas.

Consideramos que este é um excelente trabalho e que é salutar que apareça em uma revista como a RBGO, que chega a milhares de profissionais para que os ajude a entender, e compreender melhor, a problemática do planejamento familiar no Brasil e que sejam os protagonistas da mudança.

Os homens e mulheres brasileiras têm o direito constitucional de escolher quando e quantos filhos ter e o Estado tem a obrigação de fornecer os métodos, prévio conhecimento e livre eleição dos mesmos. Os direitos sexuais e reprodutivos são direitos humanos, e o acesso às ações de contracepção está dentro deste conceito. É necessário que as autoridades municipais, estaduais e federais prestem maior atenção a este problema que, além de um direito, salva a vida de milhares de mulheres.

\section{Referências}

1. Matarezi de Souza JM, Pelloso SM, Uchimura NS, de Souza F. Utilização de métodos contraceptivos entre as usuárias da rede pública de saúde do município de Maringá-PR. Rev Bras Ginecol Obstet. 2006;28(5):285-91.

2. Vieira EM, Badiani R, Fabbro ALD, Rodrigues Junior, AL. Características do uso de métodos anticoncepcionais no Estado de São Paulo. Rev Saúde Pública. 2002;36(3):263-70.

3. Faundes A, Costa RG, Pádua KS, Perdigão AM. Associação entre prevalência de laqueadura tubária e características sócio-demográficas de mulheres e seus companheiros no Estado de São Paulo, Brasil. Cad Saúde Pública. 1998; 14 Supl 1:49-57.

4. World Health Organization. Medical eligibility criteria for contraceptive use. 2nd ed. Geneva: WHO; 2004.

5. Bem-Estar Familiar no Brasil (BEMFAM). Demographic and Health Survey. Brazil: national survey on demography and health, 1996. $5^{\text {th }}$ ed. Rio de Janeiro: BENFAM/Macro International; 1997.

6. Petta CA, Faundes A, Pastene L, Pinotti JA. Users' awareness of factors associated with complications during pill use. Adv Contracept. 1994;10(4):257-64.

7. Family Health International [homepage on the Internet]. Provider checklists for reproductive health services: reference guide. 2002 [cited 2006 Feb 5]. Available from: http://www.fhi.org/en/RH/Pubs/ servdelivery/index.htm 
8. Bahamondes L, Petta CA, Faundes A, Bedone A. Significado do recente aumento do numero de solicitantes de reversão de laqueadura em um serviço de esterilidade. Femina. 1992;20(5):360-2.

9. Petta CA, Dantas C, Hidalgo MM, Bahamondes L. Solicitações de reversão da laqueadura em um serviço de esterilidade: o problema continua. Reprod Clim. 2000;15(4):214-7.

10. Petta CA, Bahamondes L, Hidalgo M, Faundes A, Bedone AJ, Faundes D. Follow-up of women seeking sterilization reversal: a Brazilian experience. Adv Contracept. 1995;11(2):157-63.

11. Hardy E, Bahamondes L, Osis MJ, Costa RG, Faundes A. Risk factors for tubal sterilization regret, detectable before surgery. Contraception. 1996;54(3):159-62.

12. Fernandes AMS, Arruda MS, Palhares MAR, Benetti-Junior ND, Moreira CM. Seguimento de mulheres laqueadas arrependidas em serviço público de esterilidade conjugal. Rev Bras Ginecol Obstet. 2001;23(2):6973.

13. Curtis KM, Mohllajee AP, Peterson HB. Regret following female sterilization at a young age: a systematic review. Contraception. 2006;73(2):205-10.

14. Hatcher RA, Rinehart W, Blackburn R, Geller JS. The essentials of contraceptive technology. Baltimore: Johns Hopkins Population Information Program; 2003.

15. Díaz J, Bahamondes L, Diaz M, Marchi N, Faundes A, Marini M. Evaluation of the performance of the copper T380A IUD up to ten years. Is this IUD a reversible but potentially permanent method? Adv Contracept. 1992;8(4):275-80.

16. Long-term reversible contraception. Twelve years of experience with the TCu380A and TCu220C. Contraception. 1997;56(6):341-52.

17. Bahamondes L, Faundes A, Sobreira-Lima B, Lui-Filho JF, Pecci P, Matera S. TCu 380A IUD: a reversible permanent contraceptive method in women over 35 years of age. Contraception. 2005;72(5):337-41. 